\title{
DOSSIER
}

\section{Producción cultural y la economía \\ en el humor digital: Observaciones de "Just for Laughs"}

Resumen

En el presente artículo, presento información relacionada a mi investigación del festival de comedia "Just for Laughs" que se llevó a cabo en Montreal, uno de los eventos más importantes que congrega actores, productores, agentes y cadenas televisoras que forman parte de la industria de la comedia. De modo particular, analizo la información de la conferencia de industria anual, "Just Comedy", la cual se llevó a cabo durante el festival. Es aquí, en que los expertos del "sector de la industria" discuten las tendencias potenciales, ansiedades y frustraciones relacionadas a la precariedad de la labor cultural y el crecimiento de la tecnología digital. Tomo en cuenta el terreno cambiante de la labor cómica, la producción del humor digital y la facilitación relacionada a las teorías de la economía creativa, la labor cultural, así como la producción cultural. La presente investigación, presenta variaciones evidentes en relación a la producción cultural sobre el crecimiento de las tecnologías digitales, así como el desempeño adecuado hacia las demandas del consumo y difusión del internet - debido a las pruebas de estándares de producción baja, comerciales cortos, y el seguimiento tanto de las masas y audiencias en particular.

Palabras clave: Humor, comedia stand-up, Internet, producción cultural, "Just for Laughs"

\section{CULTURAL PRODUCTION AND THE DIGITAL HUMOUR ECONOMY: NOTES FROM JUST FOR LAUGHS}

Danielle J. Deveau

Doctor of Philosophy candidate

Simon Fraser University

School of Communication

Master of Arts. Ryerson University, Joint

Programme in Comunication and Culture

Burnaby BC Canada 
In particular, I analyze data from the annual industry conference, Just Comedy, which takes place during the festival. Here, industry "insiders" discuss potential trends, anxieties, and frustrations related to the casualization of cultural labour and the growth of digital technologies. I consider the shifting terrain of comic labour, digital humour production, and gatekeeping in relation to theories of the creative economy, cultural labour, and cultural production. This research makes evident changes in cultural production brought about by the growth in digital technologies, as well as the suitability of stand-up performance to the demands of internet dissemination and consumption-owing largely to its amenability to low production standards, short-clips, and pursuit of both mass and specialty audiences.

Keywords: Humour, stand-up comedy, internet, cultural production, Just for Laughs.

\section{Introducción}

El festival de comedia "Just for Laughs (JFL)" es hoy en día una de las exhibiciones más grandes de la comedia stand-up en el mundo, la cual inició en 1983 como un evento francófono de comedia de cuatro días en la que se presentaron dieciséis artistas. Desde este momento, "JFL" se ha desarrollado rápidamente en el área del entretenimiento a nivel internacional de la industria. Actualmente, el festival atrae dos millones de visitantes por año, e incluye más de 350 artistas de diferentes países (en particular con la proyección en inglés y francés). Los eventos se graban y transmiten por los medios de televisión y aerolíneas en todo el mundo. Los artistas visitan las instalaciones de "JFL" a fin de lograr la conexión con los representantes de la industria del entretenimiento de Estados Unidos. Asimismo, muchas carreras se consolidan y otras culminan luego de una presentación en los escenarios de los festivales, en las secciones de la industria correspondientes a los clubes de comedias, así como en "Hyatt Lobby", en la que los medios de comunicación y artistas comentan hasta tempranas horas de la mañana.

$\mathrm{Al}$ atraer un promedio de 1000 ejecutivos de la industria, el festival se convierte en uno de los eventos más importantes para aquellas personas cuyas decisiones y preferencias determinan la dirección de la industria de la comedia. Sin embargo, se renegocia mediante el crecimiento de tecnologías digitales, el control del acceso a la información o mediante "in- 
termediarios culturales" El presente documento se basa en observaciones realizadas en "JFL" el 2008, 2009 y 2010 y, de modo particular en mi participación a la conferencia de industria anual "Just For Comedy". Aquí, los expertos de la industria, presentan sus puntos de vista en relación a los nuevos desarrollos de la industria de la comedia, de modo especial en base al crecimiento de las tecnologías digitales y el cambio en el poder de producción desde las redes a los artistas independientes. Considero, que sus discusiones académicas sobre las tendencias en la economía creativa dan poco espacio a la comedia "stand-up". Es evidente, sin embargo, que la presentación "stand up" representa una forma de producción cultural, que encaja de modo perfecto en las demandas de la difusión y consumo del internet, debido a los estándares de baja producción, comerciales cortos y el seguimiento de las masas como audiencia.

\section{La economía creativa y las prácticas laborales cambiantes}

Recientemente, consideraciones sobre producción cultural cambiaron los conceptos de "industrias creativas", así como de la "nueva economía”. El estudio de industrias creativas, combina teorías de arte creativa, industria cultural (Hartley, 2005, 6). Según John Hartley, el término industrias creativas "explota la búsqueda aproximada de los límites entre el arte creativo y la industria de la cultura”, libertad y confort, público y privado, sector comercial y ciudadano y consumidor político y personal (Harley, 2005, 18). Teniendo en cuenta en particular Nueva Zelanda, Russell Prince atribuye el rápido crecimiento de los intereses de gobierno a las "industrias creativas", al ajuste económico y la relación particular que "este" producto tiene con la fuerza de trabajo.

La explicación a esta rápida difusión implícita en la política y sus discursos de gobierno es que las economías nacionales y regionales varían en cuanto a base de industria a conocimiento de industria, innovación y creatividad - recursos que se ubican en las cabezas y cuerpos de la población que trabaja. Asimismo, menor tendencia a la fuga de capitales que ocurre cuando los recursos naturales disminuyen o los costos del trabajo se elevan. 
El recurso a desarrollar es la competencia cultural de la población. La idea de cultura como recurso es una característica de neoliberalización. George Yúdice menciona en "The Expedience of Culture" que, "la pregunta de cultura en nuestro periodo, caracterizada como una forma de globalización acelerada", se deberá enfocar como una pregunta de cultura "como recurso". Yúdice menciona que la "culturización de la economía" como parte de un cambio hacia el desarrollo cultural y componente de crecimiento económico (Yúdice, 2005, 13, 17). La cultura que cuenta en este contexto es literalmente la que se deberá tomar en cuenta de modo coherente para los accionistas dominantes; las industrias creativas se presentan como mapas estadísticos que incluyen la contribución al PBI, el número de personas que se consideran, la exportación de dólares que se gana, entre otros" (Prince, 2010, 122). Esta representación comúnmente elimina la extensión de la competencia de las industrias, de modo contradictorio y, en algunos casos, de alto nivel de explotación. Por otro lado, la narrativa de "clase creativa" que ofrecen los impulsores de la economía creativa, tales como Richard Florida, quien ofrece una narrativa convincente y conveniente para los responsables de formular la política, organizaciones de arte y ciudades que compiten por un tema de escasez de recursos e intentan realizar justificaciones económicas para el trabajo cultural.

En el marco de las industrias creativas, se justifica la inversión en cultura mediante la instrumentalización, la cual deberá estar relacionada a mejoras en las condiciones económicas, tolerancia multicultural, la promoción de la cultura de la ciudadanía, la renovación urbana o alguna otra utilidad (Yúdice, 2005, 13, 17). Yúdice sugiere que el cambio de la cultura actual depende del capital de la cultura operacional. Lo opuesto a lo que menciona Bourdieu sobre el uso metafórico de este concepto, se tiene en cuenta al capital cultural de manera literal. En relación a la organización de eventos y festivales, Jane Ali-Knight y Martin Robertson toman en cuenta el concepto de Bourdieu de modo íntegramente literal, al hablar con entusiasmo del sociólogo francés que "lideró [...] la trascendencia económica del sector cultural y del arte", un área que cuenta con un amplio potencial de mercado, que no fue tomado en cuenta por mucho tiempo overlooked (Ali-Knight and Robertson, 2004, 6). En cuanto a la economía creativa, la idea de cultura para el propósito del 
mismo, es irrelevante. El ofrecer arte, será necesario, a fin que la cultura se considere de modo serio por los responsables de formular la política, los inversionistas e incluso la audiencia.

En Firing Back, Pierre Bourdieu lamenta los impactos de neo liberalización en la vida cultural, política y social. Menciona lo siguiente bajo el concepto de neoliberalismo:

El nuevo modo de producción maximiza las utilidades al reducir la nómina mediante despidos y la compresión de salarios, concerniéndole al accionista únicamente el valor del mercado de valores, del cual depende su ingreso nominal y con estabilidad en los precios, mantiene su ingreso real lo más cerca posible al nominal. De esta forma, nos encontramos ante un régimen económico que es inseparable de un régimen político, un modo de producción que implica una forma de dominación con base en las instituciones de inseguridad, dominación a través de precariedad: un mercado financiero irregular fomenta un mercado laboral irregular y, de ese modo, la temporalidad laboral que intimida a los trabajadores para que se sometan (Bourdieu, 2003, 29).

Este mercado laboral inseguro e irregular es característico del trabajo que se realiza en la economía creativa que ha emergido bajo la globalización neoliberal. En particular, el alineamiento del trabajo creativo con el proceso de neoliberalización no representó un gran avance. El trabajo creativo ha sido por mucho tiempo inestable. El artista, con una vida laboral caracterizada por la inseguridad financiera, ya está acostumbrado a navegar en las duras realidades del trabajo ocasional, temporal y por contrato. Esto únicamente se ha intensificado a medida que las industrias creativas se han ubicado en el centro de las iniciativas de políticas neoliberales que celebran cultura y creatividad como las fuerzas impulsoras de la regeneración económica y renovación urbana (Léger, 2010, 559; Murdoch, 2003, 15).

Jamie Peck, Nik Theodore y Neil Brenner, autores de crisis financiera después del 2008, brindan una definición concisa de los procesos económicos políticos dominantes en el mundo laboral conforme al régimen financiero actual. Ellos sugieren que: 
La neoliberalización se debe concebir como un concepto de reestructuración hegemónico, como un modelo dominante de transformación reglamentaria (incompleta y contradictoria) y no como un sistema coherente completo o una forma de estado tipológico. Como tal, necesariamente opera entre sus otros entornos de gobierno múltiple, heterogéneo y contradictorio. (Peck, Theodore y Brenner, 2009, 104).

Para Peck et al, el reciente colapso financiero y la recesión económica posterior no marcan el inicio de una era posneoliberal. Esto debido a que el neoliberalismo como tal no puede declararse como si hubiese existido; en lugar de ello, el sistema económico mundial ha pasado por un proceso de liberalización que ha alterado, pero no eliminado, el papel del estado en las economías nacionales. El estado sigue participando en la "creación del mercado y en la reestructuración reglamentaria guiada por el mercado"; asimismo, la "solución" para la recesión ha sido en gran parte dar soporte y deferir a la experiencia de las instituciones que en realidad condujeron a la crisis del 2008 (Peck, Theodore y Brenner, 2009, 109). Asimismo, Kingfisher y Maskovsky argumentan que el neoliberalismo no ha sido caracterizado por un "retiro del estado", pero sí por "cambios críticos en las formas en las que los gobiernos intervienen en los mercados" (Kingfisher y Maskovky, 2008, 117). Para estos autores, el "neoliberalismo" es un modelo descriptivo útil en la medida en la que proporciona un vínculo conceptual general entre el impacto de la globalización en las economías nacionales y las justificaciones ideológicas y políticas que se han ofrecido para estos cambios dramáticos comunes (Kingfisher y Maskovky, 2008, 116). No obstante, el neoliberalismo no es "una fuerza estructural unitaria". Es contradictorio y existe en relación con muchas otras fuerzas sociales (Kingfisher y Maskovky, 2008, 119).

En este estudio de trabajo creativo, Léger señala que una fantasía determinante de neoliberalismo es la expectativa de que la economía cultural "compensarâ" los colapsos económicos de otros sectores tales como la manufactura y la agricultura (véase también Miller $e t$ ál, 2005, 116-117). Él sostiene que:

Para bien y para mal, la producción cultural, asociada con el pensamiento e innovación creativos, se ha combinado con las nuevas 
industrias, mayormente en el área de tecnología de la comunicación y se considera como un catalizador para el crecimiento económico. El nombre que los creadores de la política neoliberal le han dado a este nuevo enfoque de administración cultural es industrias creativas (Léger, 2010, 559).

El trabajo más artístico es más característico de la inestabilidad del sector de servicios (y en muchos casos, artistas que no pueden acceder a un salario base de su propio oficio al trabajar en el sector de servicios). Si el trabajo creativo está reemplazando a otras industrias como la manufactura, no se le da el mismo nivel de estabilidad y predictibilidad que esas industrias ofrecen a su personal. El trabajador cultural es un emprendedor creativo, cuyo movimiento precario dentro de la industria es difícil de identificar y registrar. Jan Marontate y Catherine Murray han sostenido que esta característica del trabajo creativo genera la formulación de la política para el soporte de los trabajadores que cuestionan ideas establecidas:

No es una pequeña parte debido a la naturaleza de las empresas de negocios en estos campos, marcadas por cambios constantes, variaciones en la demanda de la temporada y modelos de empleo que implican el empleo a medio tiempo de forma concomitante e intermitente, además de la participación de personal voluntario importante (Marontate y Murray, 2010, 333).

De este modo, los trabajadores culturales se han encontrado en una posición curiosa en la que se ha promocionado considerablemente su campo como la clave de una economía próspera, siendo sus condiciones laborales más precarias de lo que fueron antes (Léger, 2010; Brault, 2010; Hesmondalgh y Baker, 2010). Este es el incentivo principal para que las comedias en vivo salgan de las presentaciones en vivo y se vayan a las "industrias culturales", tales como el cine y la televisión. Sin embargo, este trabajo también es cada vez más impredecible y por contratos. Con el crecimiento de las tecnologías digitales, los nuevos enfoques, centrados en lo humorístico, de la producción y difusión se están apropiando de la industria. En particular, la producción cultural de hágalo 
usted mismo hizo posible, a través de equipos baratos de video, software de edición y sitios de transmisión rápida y gratuita como youtube.com, la desestabilización no solo de la creación de la comedia, sino también los reembolsos por el trabajo. En Just Comedy 2008, 2009 y 2010, estos problemas fueron ampliamente discutidos y debatidos por las "personas que pertenecen a la industria", incluidos ejecutivos de la red, productores, escritores y actores. A continuación presento hallazgos de datos iniciales recolectados en este periodo de tres años mientras realizaba una observación naturalista en JFL.

\section{Las condiciones en la producción de comedias}

\section{Métodos de investigación}

Durante julio de 2008, 2009 y 2010, visité Montreal para realizar una observación de JFL. Uno de los espacios más ricos en datos de este evento probó ser Just Comedy, un congreso anual de la industria que se llevó a cabo en Hyatt Regency (actualmente la sede de la logística de JFL durante el festival). Durante el evento, asistí a presentaciones, discursos y diversas reuniones de la industria. Los datos con los cuales redacté este documento forman parte de notas de campo tomadas, especialmente de los paneles que tratan de condiciones laborales cambiantes y el impacto de Internet en la producción humorística. En particular, diversos paneles que no estaban previstos para tratar estos temas lo hicieron ya sea durante el panel, o en la ronda de preguntas. Así que se evidenció que los asistentes al congreso estaban muy preocupados por estos temas.

\section{Considerando los discursos de la industria en Just Comedy}

Esta sección presentará datos obtenidos del congreso con respecto a tres asuntos principales: 1) el cambio en las condiciones laborales en la producción humorística; 2) el impacto de las tecnologías digitales en la producción y difusión de contenido y 3) el terreno cambiante del control en la era de Internet. 


\section{Mano de obra}

La mano de obra es un asunto particularmente polémico en la producción cultural debido a que las industrias de cine y televisión estadounidenses y canadienses tienen una larga historia de sindicalización, mientras que las industrias de la comedia en vivo no la tienen. Como tal, se deben esperar que los actores, escritores y productores con distintos antecedentes en la industria tengan visiones divergentes sobre la mano de obra industrial, especialmente con relación al trabajo creativo no remunerado. Por ejemplo, en la industria de la comedia en vivo canadiense se puede esperar que los actores participen en trabajos no remunerados o poco remunerados al inicio de sus carreras para que desarrollen habilidades y un perfil de comediante (Stebbins, 1990). Estos primeros ańos precarios y explotadores son parte de una ideología industrial mayor que indica que los actores jóvenes deben "pagar su derecho a piso" antes de ser aceptados en una clase de actores profesionales.

Dentro de este sistema, se ve a Internet como otro medio de desarrollo de perfil disponible para los escritores y actores. Esta fue con seguridad la perspectiva propuesta por múltiples panelistas en el congreso de 2008. Lou Wallach, Vicepresidente Sénior de Desarrollo Digital y Tecnológico, de Televisión y Programación Original de Comedy Central sugirió que Internet ofrece un espacio vital de desarrollo, un buen lugar para que el trabajo se vea y una forma de "abrirse paso" en las redes de televisión (Panel de Webisodio a Episodio, 17 de julio de 2008). En otro panel para la discusión de la difusión, tanto Michelle Daly, Directora de Contenidos de Comedy Network como Anton Leo, Jefe Creativo de TV Comedy en CBC/Radio Canadá manifestaron que Internet ofrece un espacio invalorable para el desarrollo y descubrimiento de nuevos talentos. No obstante, también dijeron que es poco probable que sus redes puedan obtener dinero del contenido en línea (Panel de difusión, 17 de julio de 2008). Como tal, se espera que el contenido de internet sea una manera marginal de difusión de contenido con redes que esperan que las audiencias de la web se redirijan a su programación de difusión. Los escritores, actores y productores que esperan ingresar a la industria de la comedia pueden utilizarla para difundir su material, pero se espera que estos trabajadores culturales necesiten integrarse en las industrias de cine 
y televisión establecidas para que puedan vivir de su trabajo cultural. Al siguiente año, Renate Radord, Vicepresidente de Comedy Programming para NBC Entertainment \& Universal Media Studios, admitió que los difusores aún no tenían claro de qué forma se podría obtener dinero a partir de la programación en Internet.

Esta perspectiva también se promocionó en el congreso del 2010. Esto incluyó a un panel completo de FunnyorDie.com (FOD), un sitio web de comedia por Internet muy exitoso que produce contenido original, reproduce videos humorísticos virales y brinda a los miembros de su comunidad digital la oportunidad de difundir su propio trabajo humorístico. Los videos producidos por el personal de FOD, por lo general, incluyen actuaciones especiales de celebridades (músicos, comediantes, actores populares, etc.). Durante el panel, el actor/escritor de FOD, Seth Morris, sostuvo que una vez que pueda obtener dinero del contenido de Internet, la "era dorada" de la producción de la comedia digital llegará a su fin. FOD se beneficia en gran parte de producción de contenido no remunerada ni regulada. Cuando un miembro de la audiencia le preguntó al panel sobre la ética de utilizar mano de obra no remunerada de personal de otro modo sindicalizado, los panelistas indicaron que cualquier intrusión de sindicalización en el tipo de producción que estaban haciendo haría que su trabajo creativo sea imposible. Sin embargo, los panelistas no vieron esto como una forma de explotación, pero sí como un espacio de producción que permite comedia pura, libre del impacto de la censura de la economía cultural.

\section{Producción digital}

Como un espacio de desarrollo de talentos y contenido, existe evidencia de que Internet va a continuar. En el panel de Webisodio a Episodio (2008), Peter Oldring trató algunas de las ventajas y desventajas de esta trayectoria. Su parodia de TV matutina: "Good Morning World" ("Buenos Días al Mundo") se estableció como un webisodio. Como tal, se produjo con bajo presupuesto, tuvo poco tiempo de desarrollo y se alojó en un simple sitio de Internet. Una vez que este programa fue producido por la red, el comediante tuvo que hacer algunos cambios menores (acorde con las expectativas de producción de la red), pero, en 
general, Oldring siente que sus ideas originales y personajes permanecen intactos. John Gemberling y Curtis Gwinn también contaron con webisodios en la red. Ellos produjeron su programa original: "Fat Guy Stuck in the Internet" ("El Gordito Pegado a Internet"), con base en lo que ellos consideraban que era divertido. Ellos no buscaron soporte de la red, pero hallaron la forma de conseguir un trato luego de que su programa se descubrió en línea por un ejecutivo interesado en el mismo. A pesar de la mejora en el valor de la producción que la asociación de la red entregó, Gemberling y Gwinn fueron optimistas en cuanto a que la TV continuaría en línea. En estos dos ejemplos, los escritores/actores no descubiertos lo fueron a través de contenido en línea autofinanciado y autoproducido. Esto refuerza la idea de que, aunque no sea directamente lucrativo para el productor de comedia en línea, Internet es un espacio efectivo para el desarrollo del material y del perfil.

\section{Controles}

Los avances en las tecnologías de comunicación global también tienen un impacto en el balance de las fuerzas entre los controladores dominantes en el campo. Por ejemplo, en los últimos dos años en Just Comedy, un asunto ha dominado la conversación y ha estado presente en casi todos los paneles, independientemente del tema a tratar: Internet. La siguiente pregunta conlleva una preocupación particular: ¿Internet acabará con la televisión abierta? De no ser así, ¿cómo las redes podrán obtener dinero del contenido de Internet? Parecería que el modelo estándar de la comedia en la televisión abierta se estaría sometiendo a evaluación. Los productores de contenido han podido evitar los procesos de riesgo de lanzar conceptos pilotos a las redes principales, optando en lugar de ello por crear videos con bajo valor de producción que se puedan publicar en internet. Una vez que un video es difundido, los creadores esperan que sea tomado por alguna red o, con mayor probabilidad, por un canal por cable especializado. Parece irrazonable continuar invirtiendo en recursos para la producción de pilotos cuando los nuevos contenidos de comicidad se encuentran constantemente disponibles de forma gratuita. Además, la comedia empresarial puede superar a muchos con- 
troladores establecidos como los clubes y festivales de la comedia a través del contenido en línea, conectándolos directamente con una audiencia masiva. En la actualidad, no existe evidencia de que las oportunidades de "éxito" como productor próspero de contenido de Internet sean necesariamente mejores que las de los actores que optan por los sistemas establecidos en las industrias culturales de controladores. Internet está llena de contenido que recibe poca o ninguna atención. Sin embargo, el contenido que se encuentra en gran circulación en sitios tales como FunnyOrDie o YouTube llegará a cantidades de audiencia que muchas redes -debido a que el crecimiento de la televisión por cable ha separado a la audiencia masiva- ya no podrán lograr. Esto no es para exagerar el impacto que la comedia por Internet haya tenido en el consumo televisivo. Varios de los controladores tradicionales han crecido para tomar ventaja de esta nueva forma de difusión de contenido.

El contenido de Internet tiene un valor de producción bajo y ha limitado el desarrollo de la narración. Se basa en personajes únicos en situaciones humorísticas. Los videos más exitosos son los que tienen una duración de unos pocos minutos o incluso segundos. Este tipo de contenido de Internet se ha vuelto esencialmente una forma de "desperdiciar tiempo en el trabajo" (Seth Morris, Panel de FunnyorDie, 16 de julio de 2010). El énfasis en producciones de alto costo que es, por lo general, característico de la televisión abierta simplemente no se aplica al contenido de Internet en el que los archivos de medios de alta definición son difíciles de visualizar dependiendo de su velocidad. La generación de Internet ha demostrado que la comedia de calidad es importante para ellos, pero el valor de la producción se puede ver sacrificado a cambio de facilidad de acceso y circulación. Los géneros de comedia en vivo y sketch son en particular muy adecuados para programación por Internet ya que tienden a ser consumidos en "clips" de corta duración y su disfrute no depende en gran parte de un alto valor de producción o la calidad de la imagen.

\section{Conclusión}

El crecimiento de las tecnologías digitales ha cambiado la forma en la que los actores de comedia son descubiertos, así como algunas de las for- 
mas en las que el contenido cómico se produce y difunde. Los cómicos están utilizando la producción del contenido de internet gratuito, por lo general no remunerado y autofinanciado para el desarrollo de sus perfiles y ganar el pase a las industrias culturales tan controladas. Este tipo de trabajo precario y poco remunerado es por lo general característico de la economía creativa. Sin embargo, los cómicos también se benefician de este nuevo tipo de producción al evitar a algunos de los controladores tradicionales en el campo. Esto permite que los actores adquieran mayor control creativo en su trabajo y en sus carreras. La digitalización de la industria de la comedia está alterando la forma en la que se produce humor, así como el sistema de producción de talento cómico. Estos cambios están ocurriendo rápidamente y tienen todavía impactos ilimitados en la forma y dirección en el campo de la comedia.

\section{Referencias}

Ali-Knight, J. y Robertson, M. (2004) Introduction to Arts, Culture and Leisure. (Introducción a las Artes, Cultura y Entretenimiento). En Festival and Events Management: An International Arts and Culture Perspective. (Gestión de Eventos y Festivales: Una Perspectiva Internacional de Artes y Culturas). I. Yeoman et ál., Ed. Elsevier, Boston, 3-13.

Bourdieu, P. (2003) Firing Back: Against the Tyranny of the Market 2. (Contraatacando: Contra la Tiranía del Mercado 2). Verso, Londres.

Brault, S. (2010) No Culture, No Future. (Sin Cultura, Sin Futuro). J. Kaplansky, Trans. Cormorant Books, Toronto.

Hartley, J., Ed. (2005) Creative Industries. (Industrias Creativas). Blackwell, Oxford.

Hesmondalgh, D., y Baker, S. (2010) 'A very complicated version of freedom': Conditions and experiences of creative labour in three cultural industries. ('Una Versión muy Complicada de Libertad': Condiciones y Experiencias de Trabajadores Creativos en Tres Industrias Culturales). Poetics. 38, 4-20.

Kingfisher, C., y Maskovsky, J. (2008). The limits of neoliberalism. Critique of Anthropology. (Los Límites del Neoliberalismo. Crítica de Antropología). 28(2), 115-126. 
Léger, M.J. (2010) The non-productive role of the artist: The creative industries in Canada. Third Text. (El Papel No Productivo del Artista: Las Industrias Creativas en Canadá. Tercer Texto). 24(2), 557-570. Marontate, J. y Murray, C. (2010) Neoliberalism in Provincial cultural policy narratives: Perspectives from two coasts. (El Neoliberalismo en la Narrativa de la Política Cultural Provincial: Perspectivas desde Dos Costas). Canadian Journal of Communication. (Revista Canadiense de Comunicación). 35, 325-343.

Miller, T., et ál. (2005) Global Hollywood 2. (Hollywood Global 2). British Film Institute, Londres.

Murdoch, G. (2003) Back to work: Cultural labor in altered times. (De Vuelva al Trabajo: Trabajadores Culturales en Tiempos Alterados). En Cultural Work: Understanding the Cultural Industries. (Trabajo Cultural: Entendiendo a las Industrias Culturales). A. Beck, Ed. Routledge, Londres, 15-36.

Peck, J., Theodore, Nik., Brenner, N. (2009) Postneoliberalism and its malcontents. (El Posneoliberalismo y sus Descontentos). Antipode 41(1), 94-116.

Prince, R. (2010) Globalizing the creative industries concept: Travelling policy and transnational policy communities. (Globalizando el Concepto de las Industrias Creativas: Comunidades de Políticas Transnacionales y Políticas de Viaje). The Journal of Arts Management, Law, and Society. (Revista de Gestión de Artes, Derecho y Sociedad). 40, 119-139.

Stebbins, R. (1990) The Laugh-Makers: Stand-Up as Art, Business, and Life-Style. (Los Hace Risas: Como Arte, Negocio y Estilo de Vida). McGill-Queen's UP, Montreal.

Yudice, G. (2005) The Expediency of Culture: Uses of Culture in the Global Era. (La Conveniencia de la Cultura: Usos de la Cultura en la Era Global). Duke UP, Durham y Londres.

Correo electrónico: ddeveau@sfu.ca 